\title{
Ultrafast Molecular Frame Electronic Coherences from Lab Frame Scattering Anisotropies
}

\author{
Varun Makhija, ${ }^{1,2, *}$ Kevin Veyrinas, ${ }^{1}$ Andrey E. Boguslavskiy, ${ }^{1,3,4}$ \\ Ruaridh Forbes, ${ }^{1,5}$ Iain Wilkinson, ${ }^{4,6}$ Rune Lausten, ${ }^{4}$ Simon P. Neville, ${ }^{3}$ \\ Stephen T. Pratt, ${ }^{7}$ Michael S. Schuurman, ${ }^{3,4}$ and Albert Stolow ${ }^{1,3,4, \dagger}$ \\ ${ }^{1}$ Department of Physics, University of Ottawa, \\ 150 Louis Pasteur, Ottawa, ON, K1N 6N5, Canada \\ ${ }^{2}$ Department of Physics, University of Mary Washington, \\ Fredericksburg, Virginia 22401, USA \\ ${ }^{3}$ Department of Chemistry, University of Ottawa, Ottawa, ON, K1N 6N5, Canada \\ ${ }^{4}$ National Research Council of Canada, \\ 100 Sussex Drive, Ottawa, ON, K1N 5A2, Canada \\ ${ }^{5}$ Department of Physics and Astronomy, University College London, \\ Gower Street, London, WC1E 6BT, UK \\ ${ }^{6}$ Locally-Sensitive $\&$ Time-Resolved Spectroscopy, \\ Helmholtz-Zentrum Berlin für Materialien und Energie, \\ Hahn-Meitner-Platz-1, 14109 Berlin, Germany \\ ${ }^{7}$ Chemical Sciences and Engineering Division, \\ Argonne National Laboratory, Lemont, IL, 60439, USA
}

\begin{abstract}
Electronic coherences in molecules are ultrafast charge oscillations on the Molecular Frame (MF) and their direct observation and separation from electronic population dynamics is challenging. Here we present a valence shell Lab Frame (LF) scattering method suited to probing electronic coherences in isolated systems. MF electronic coherences lead to LF electronic anisotropies observable by ultrafast angle-resolved scattering. Moment analysis of the LF anisotropy completely separates electronic coherences from population dynamics, demonstrated in excited state $\mathrm{NH}_{3}$ using ultrafast time-energy-angle-resolved photoelectron spectroscopy. This general approach applies equally to attosecond/femtosecond electronic coherences in isolated systems.
\end{abstract}

\footnotetext{
*vmakhija@umw.edu

† astolow@uottawa.ca
} 


\section{INTRODUCTION}

Electronic coherences in molecular systems underlie their structural and dynamical properties. In attosecond science, coherences prepared by ultrashort laser pulses create electronic wavepackets [1-4]. On longer time scales, atomic motions modify these coherences, leading to dephasing and electronic population dynamics $[3,5,6]$. Nuclear motion also induces and alters electronic coherences and populations via non-adiabatic couplings [7-11]. In polyatomics, electronic population dynamics at conical intersections [12] can be probed using techniques such as Time-Resolved Photoelectron Spectroscopy [13-17]. A key challenge is the direct observation of electronic coherences and their separation from electronic population dynamics $[6,7,18-20]$, requiring observables such as the nonlinear X-ray spectroscopies theoretically proposed for observing electronic coherences induced by passage through a conical intersection [7-9]. Here we propose and demonstrate a general wavepacket approach, based on angular momentum correlations in isolated systems, which completely separates electronic population dynamics from electronic coherences. We emphasize two points: (i) Molecular Frame (MF) electronic coherences are manifested as Lab Frame (LF) anisotropies (electronic probability distributions) of the ensemble; (ii) angular momentum correlations permit the complete separation of coherences from population dynamics. LF electronic anisotropies are characterized by their moments, such as orientation and alignment: their time dependence directly tracks MF electronic coherences.

In Fig. 1(a), we depict the coherent preparation of a molecular electronic (rovibronic) wavepacket by a resonant ultrashort pump pulse. Wavepacket dynamics are observed by projection onto a final (e.g. ionic) state using an ultrashort probe pulse (not shown). Interference between degenerate probe transitions from pairs of coherent eigenstates leads to quantum beats with periods determined by the level spacings involved: the sum of all such beats comprises the wavepacket signal. Consider two electronic states $|n\rangle$ and $\left|n^{\prime}\right\rangle$ with energy gap $\Delta E_{\text {elec }}$, each with vibrational and rotational $(J$, MF projection $K$ ) states.

Typically, 'dark' states (not shown) non-adiabatically couple to $|n\rangle$ and/or $\left|n^{\prime}\right\rangle$, leading to electronic population dynamics. LF anisotropy induced by MF electronic wavepacket dynamics is understood in terms of the wavepacket's angular momentum composition. Of the different angular momentum coherences in Fig.1(a), we emphasize those $(\Delta J=2)$ which generate higher moments of the LF anisotropy. These, we show, allow complete separation 
(a)

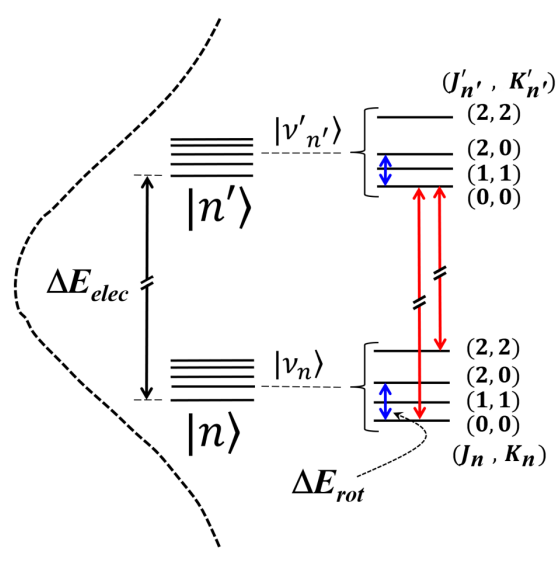

(b)

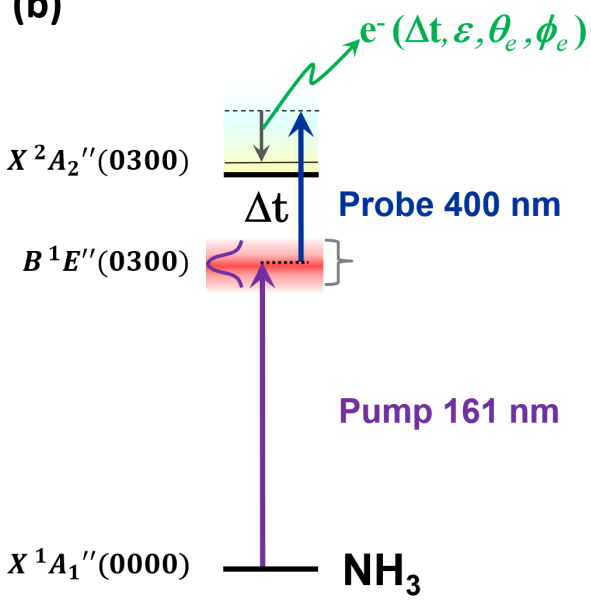

(c)

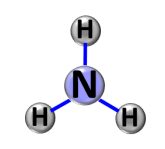

$\Delta \mathrm{t}$

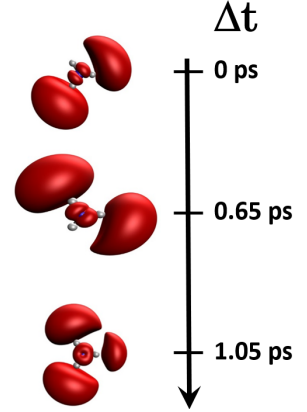

FIG. 1. (a) Two electronically excited states $|n\rangle$ and $\left|n^{\prime}\right\rangle$ with bandgap $\Delta E_{\text {elec }}$ are shown, each having their associated vibrational and rotational states. The dashed line indicates a resonant ultrashort laser pulse. For details, see the text. (b) A depiction of the pump-probe TRPADs scheme used in the experiment. The pump bandwidth (red) coherently prepared the two electronic states which arise from the doubly degenerate $\tilde{B}^{1} E^{\prime \prime}$ state of $\mathrm{NH}_{3}$. (c) A calculation of the evolving MF electronic probability distribution for coherences initiated by pumping from the $\left(J_{0}, K_{0}\right)=(1,1)$ ground rotational state (see SM for details). The time evolution shown is for one of the dominant frequency components observed in the experiment $\left(23 \mathrm{~cm}^{-1}\right.$ Fig. 5(c)). This coherence, shown at time delays $\Delta t=0,0.65$ and $1.05 \mathrm{ps}$, corresponds to the upper red arrow in (a).

of the electronic population dynamics from coherences. Dipole selection rules always lead to the coherent preparation of $\Delta J=2$ pairs in each of the excited electronic states - the $P$ and $R$ spectroscopic branches [21]. We classify these coherences as to whether they are within a single electronic state (be it $|n\rangle$ or $\left|n^{\prime}\right\rangle$ ), called intrastate coherences (blue arrows), or are between electronic states, called interstate coherences (red arrows). The intrastate angular momentum coherences are well known and form the basis of rotational coherence spectroscopy [22], evolving on rotational time scales (the inverse of $\Delta E_{\text {rot }}$ ). These track the rotational dynamics within each electronic state. The interstate electronic coherences evolve on time scales largely governed by the electronic gap, $\Delta E_{\text {elec }}$. For typical valence electronic states, $\Delta E_{\text {elec }}$ is much larger than $\Delta E_{\text {rot }}$. The way in which these different MF angular momentum coherences are manifested and separated in the ensemble-averaged LF electronic anisotropy is our first key point. Regardless of their origin, MF electronic coherences re- 
sult in a LF electronic anisotropy evolving on time scales determined by the MF dynamics. Our second key point is that any ultrafast angle-resolved LF scattering measurement, be it electron or X-ray scattering or angle-resolved photoemission, is a selective probe of this evolving LF electronic anisotropy and, thus, the electronic coherences. Here we demonstrate that Time-Resolved Photoelectron Angular Distributions (TRPADs) completely separates electronic population dynamics from electronic coherences and, to a high degree, further separates rotational from electronic coherences. At conical intersections, electronic level spacings $\Delta E_{\text {elec }}$ become comparable to vibrational level spacings. Since the latter are generally larger than $\Delta E_{\text {rot }}$, time scales alone will separate rotational from electronic coherences, suggesting that our method will be well suited to probing electronic coherences at conical intersections.

To illustrate, we excited electronic population dynamics and coherences in the $\mathrm{NH}_{3} \tilde{B}^{1} E^{\prime \prime}$ state, probed by time-delayed single photon ionization. Here, electronic population dynamics are due to non-adiabatic coupling to the dissociative $\tilde{A}^{1} A_{2}^{\prime \prime}$ state, whereas the electronic coherences are due to non-adiabatic rotational (Coriolis) and vibrational (Jahn-Teller) interactions. The electronic Coriolis dynamics provides the physically intuitive picture of the MF electronic wavepacket 'not keeping up' with MF rotation (see Fig.1(c)). However, in $\mathrm{NH}_{3}$ the electronic bandgap $\Delta E_{\text {elec }}$ is unusually small, since it is due to the electronic Coriolis effect: the two electronic states $|n\rangle$ and $\left|n^{\prime}\right\rangle$ have a $\Delta E_{\text {elec }}$ which is on the same order as $\Delta E_{r o t}$. This is the most challenging test of our method because, although population dynamics are completely separable via angular momentum correlations (vide infra), the rotational and electronic coherences are not separable here by time scale alone, as would be the case for most valence state electronic wavepackets. Nevertheless, we demonstrate complete separation of populations from coherences with good separation of rotational (intrastate) from electronic (interstate) coherences.

In Section II, below, we describe how the LF charge distribution is sensitive to MF electronic dynamics. This leads to the definition of new time dependent quantities - the Electronic Angular Distribution Moments (EADMs) - which transduce MF electronic coherences into the LF. In Section III, we describe the separation of electronic coherences from population dynamics facilitated by measurement of the EADMs. This section is further divided into three subsections. Section A describes the experimental method of Time Resolved Photoelectron Angular Distributions (TRPADs) used here. Section B describes the 
theoretical model used to calculate the EADMs in the $\tilde{B}^{1} E^{\prime \prime}$ state of $\mathrm{NH}_{3}$. This section also describes in detail the well known spectroscopy of the $\tilde{B}^{1} E^{\prime \prime}$-state. Section III C presents the experimental results, and discusses their interpretation based on the general concepts presented in Section I. This section also briefly describes the photoionization formalism developed in [23], which can be used to to describe the measurable quantities in terms of EADMs. Finally, Section IV summarizes our main results in the context of attosecond measurements in isolated molecules, and suggests future direction based on our findings.

\section{THE LABORATORY FRAME CHARGE DISTRIBUTION}

In the MF, an electronic coherence generates a time-dependent electronic charge distribution having MF spherical multipole moments $\gamma_{S}^{K}$ with time-dependent expectation values. Here $K=0,1,2 \ldots$ are, respectively, the monopole, dipole, quadrupole etc. moments of the MF charge distribution, with spherical components $S$ ranging from $-K$ to $K$. The time dependence of the LF multipoles, $\Gamma_{Q}^{K}$, obtains from a simple rotation into the LF [24],

$$
\Gamma_{Q}^{K}=\sum_{S} D_{Q S}^{K *}(\Omega) \gamma_{S}^{K}
$$

The $D_{Q S}^{K *}(\Omega)$ are the Wigner rotation matrix elements with Euler angles $\Omega=\phi, \theta, \chi$. For an ensemble, the time dependent expectation value of $\Gamma_{Q}^{K}$ is constructed from the density matrix, here in a basis of separable vibrational-electronic (vibronic) and rotational states, $|n\rangle$ and $\left|J_{n} K_{n} M_{n}\right\rangle$, respectively. $J_{n}$ is the angular momentum in vibronic state $|n\rangle$, with projections $K_{n}$ and $M_{n}$ onto MF and LF quantization axes, respectively. While $n$ is a general vibronic state label, we restrict its use here to electronic states, as in Fig. 1(a). In this basis,

$$
\begin{aligned}
\left\langle\Gamma_{Q}^{K}\right\rangle(t)= & \sum_{n} \sum_{S}(-1)^{(K+S)} A_{Q-S}^{K}(n, n ; t)\left\langle n\left|\gamma_{S}^{K}\right| n\right\rangle \\
& +\sum_{n \neq n^{\prime}} \sum_{S}(-1)^{(K+S)} A_{Q-S}^{K}\left(n, n^{\prime} ; t\right)\left\langle n^{\prime}\left|\gamma_{S}^{K}\right| n\right\rangle .
\end{aligned}
$$

Note that we have deliberately broken this sum into two, one in which the electronic index is preserved $\left(n=n^{\prime}\right)$ and one is which it is different $\left(n \neq n^{\prime}\right)$. As discussed in detail below, this separation facilitates the distinction of rotational coherences $\left(n=n^{\prime}\right)$ from electronic coherences $\left(n \neq n^{\prime}\right)$. The time dependence of the LF anisotropy is entirely contained in the quantities $A_{Q S}^{K}(n, n ; t)$ and $A_{Q S}^{K}\left(n, n^{\prime} ; t\right)$. These were previously defined in the formalism for molecular photoionization developed by Stolow and Underwood [23] as follows, 


$$
\begin{aligned}
& A_{Q S}^{K}\left(n, n^{\prime} ; t\right)=\sum_{J_{n} K_{n} M_{n}} \sum_{J_{n^{\prime}}^{\prime} K_{n^{\prime}}^{\prime} M_{n^{\prime}}^{\prime}} \sqrt{\left(2 J_{n}+1\right)\left(2 J_{n^{\prime}}^{\prime}+1\right)}(-1)^{K+J_{n}+J_{n^{\prime}}^{\prime}-\left(M_{n}+K_{n}\right)} \\
& \times\left(\begin{array}{ccc}
J_{n} & J_{n \prime}^{\prime} & K \\
M_{n} & -M_{n^{\prime}}^{\prime} & -Q
\end{array}\right)\left(\begin{array}{ccc}
J_{n} & J_{n^{\prime}}^{\prime} & K \\
-K_{n} & K_{n^{\prime}}^{\prime} & S
\end{array}\right) \rho_{\omega_{n}, \omega_{n^{\prime}}^{\prime}}\left(n, n^{\prime}\right)(t) .
\end{aligned}
$$

Here $\rho_{\omega_{n}, \omega_{n^{\prime}}^{\prime}}(t)\left(n, n^{\prime}\right)$ is the time dependent density matrix, and the index $\omega_{n}$ collectively represents the angular momentum quantum numbers in the electronic state $n$. The terms in large parentheses represent Wigner 3j symbols which combine each pair of angular momenta $J_{n}, J_{n^{\prime}}^{\prime}$ in the wavepacket resulting in the total angular momentum $K=\left|J_{n}-J_{n^{\prime}}^{\prime}\right|, \mid J_{n}-$ $J_{n^{\prime}}+1|, \ldots,| J_{n}+J_{n^{\prime}}-1|,| J_{n}+J_{n^{\prime}} \mid . Q$ and $S$ are the LF and MF projections, respectively, of $K$ and in each case range from $-K$ to $K$.

The sum in Eq. 2 first is over states preserving the electronic index (whether $n$ or $n^{\prime}$ ), reflecting the intrastate coherences (blue arrows) in Fig.1(a). The second sum is over states with differing electronic indices, reflecting the interstate coherences (red arrows). The intrastate coherences $A_{Q S}^{K}(n, n ; t)$ are the well known Axis Distribution Moments (ADMs) for each electronic state $|n\rangle$ or $\left|n^{\prime}\right\rangle \quad[23,25]$, as studied in rotational coherence spectroscopy. The rotational evolution of the molecular axis distribution in each electronic state can be expressed in the LF via the ADMs through $P(\Omega, t)=\sum_{K Q S} A_{Q S}^{K}(n, n ; t) D_{Q S}^{K *}(\Omega)[23,25]$. Thus, the first sum in Eq. 2 describes the LF anisotropy moments which evolve as does the MF axis distribution: these intrastate coherences result in a MF charge distribution which appears stationary in the rotating frame. ADMs in isolated molecules were previously studied by time-resolved photoionization using both perturbative [26-35] and strong laser fields [35-37]. In the second sum, the interstate coherences $A_{Q S}^{K}\left(n, n^{\prime} ; t\right)$ have an entirely different, hitherto unexplored physical meaning not interpretable as a distribution of MF axes. These are the dynamic, MF electronic contributions to $\left\langle\Gamma_{Q}^{K}(t)\right\rangle$ which evolve in the rotating frame $(\mathrm{MF})$ : they are the $\mathrm{MF}$ electronic coherences with frequecies determined by $\Delta E_{\text {elec }}$. What is their relationship to the LF electronic anisotropy? The ensemble averaged multipoles $\left\langle\Gamma_{Q}^{K}\right\rangle(t)$ quantify the LF electronic alignment (even $K$ ) and orientation (odd $K$ ). Thus, the first sum in Eq. 2 is the LF electronic anisotropy due to MF axis alignment/orientation and evolves on rotational timescales according to $\Delta E_{\text {rot }}$. The second sum is the LF electronic anisotropy due to electronic coherences between states $n$ and $n^{\prime}$ and evolves on electronic timescales according to $\Delta E_{\text {elec }}$. To distinguish their interpretation, we 
term the $A_{Q S}^{K}\left(n, n^{\prime} ; t\right)$ as Electronic Angular Distribution Moments (EADMs): these encode MF electronic coherences into the LF electronic anisotropy, our first key point. Finally, we note that the $K=0 \mathrm{ADMs} / \mathrm{EADMs}$ map directly to the reduced electronic density matrix $\rho\left(n, n^{\prime}\right)(t)$. From Eq. (2) we see that these only affect the isotropic LF electronic moment $\left\langle\Gamma_{0}^{0}\right\rangle(\mathrm{t})$, and thus cannot contribute to the corresponding LF electronic anisotropy. Therefore, the electronic populations $\rho(n, n)(t)$ are excluded from the LF anisotropy, and relegated to the isotropic monopole moment. The LF electronic anisotropy therefore results only from electronic coherences between angular momentum states with $J_{n} \neq J_{n^{\prime}}^{\prime}$.

It is worth noting that what may be interpreted as population transfer in one basis set, may appear as coherences in another [38, 39]. Nonetheless, the ADMs and EADMs described here may still be computed using Eq. 3, since $J$ is always a conserved quantity for an isolated system. This feature also ensures that the physical interpretation of these quantities holds regardless of basis set choice: this ensures that the ADMs and EADMs may be used generally to interpret the results of an experiment in an isolated system. The particular choice of basis set in any given case is usually motivated by the particular set of states onto which the wavepacket is projected [38-40]. In the next section, we discuss the separation of electronic coherences from population dynamics in the adiabatic basis, since this is the basis of interest when discussing passage through a conical intersection.

We further emphasize that the above conclusions are independent of the intensity of the pump (preparation) laser pulse. The method (e.g. strong or weak laser pulses, electron pulses) used to excite the wavepacket is irrelevant. It was previously shown that strong-field probes are sensitive to interstate and intrastate coherences [3, 5, 41-43], making such experiments suitable for analysis using the general ADM and EADM formalism developed here. Below we specifically discuss the case of one-photon ionization of the excited wavepacket, as is typically the case for the well established pump-probe TRPES technique used here. .

\section{SEPARATION OF ELECTRONIC COHERENCES AND POPULATION DY- NAMICS}

Our second key point follows directly from above: any observable selective of the electronic anisotropy or, in other words, selective of EADMs with $K>0$, will separate electronic coherences from population dynamics. We experimentally demonstrate here that the highest 
moment of a LF scattering anisotropy does exactly this for electronic-nuclear wavepackets in $\mathrm{NH}_{3}$, in this case using ultrafast energy-angle-resolved TRPADs as the scattering technique. Since this fact derives from the conservation of angular momentum, it can be generalized to any angle-resolved ultrafast scattering experiment. In sections B and C below, we describe the relevant experimental and theoretical methods used to achieve and interpret our results.

\section{A. Experimental Method}

Fig 1(b) depicts the sequence of events in the experiment. A $160.9 \mathrm{~nm}, 77 \mathrm{fs}$ pump pulse resonantly excited $\mathrm{NH}_{3}$ into two closely spaced electronic states arising from the degenerate $\tilde{B}^{1} E^{\prime \prime}$ state. The optical setup and Velocity Map Imaging (VMI) spectrometer used in this experiment were previously described in detail [44]. Briefly, $3.25 \mathrm{~mJ}$ of a $7.5 \mathrm{~mJ}, 35 \mathrm{fs}$, $800 \mathrm{~nm}$ laser pulse was used to generate pump and probe pulses for the experiment. $2.5 \mathrm{~mJ}$ was used to generate a $160.9 \mathrm{~nm}$, approximately 77 fs pump pulse by four-wave mixing with the third harmonic of $800 \mathrm{~nm}$ in argon gas [45, 46]. This is combined on a dichroic highreflector with a $20 \mu \mathrm{J}, 400 \mathrm{~nm}, 40$ fs probe pulse generated by second harmonic generation in a BBO crystal. The pulses were spatio-temporally overlapped and focused by a spherical reflective mirror into a VMI spectrometer, where they intersected a $1 \mathrm{kHz}$ pulsed molecular beam generated by expansion of $2.1 \% \mathrm{NH}_{3}$ seeded in Helium through an Even-Lavie valve, and skimmed through a $1 \mathrm{~mm}$ diameter skimmer. The stagnation pressure of the $\mathrm{NH}_{3}-\mathrm{He}$ mixture was set at 20 psi. This condition was determined by time-of-flight mass spectrometry at several stagnation pressures and pulsed valve timings in order to yield conditions which minimized the contribution of clusters. These experiments were performed under conditions in which the contribution of clusters was negligible.

The $160.9 \mathrm{~nm}$ pump pulse resonantly excited $\mathrm{NH}_{3}$ to the planar $B^{1} E^{\prime \prime}$ electronic state. The time delayed $400 \mathrm{~nm}$ probe pulse then photoionized the excited state molecule and the kinetic energy spectrum and angular distribution of the ejected electron were measured as a function of time delay using the VMI spectrometer. Data was collected between -0.3 and 5 ps with a step size of 0.04 ps. The data presented here result from an average over 20 such delay scans.

Fig. 2 (a) shows the time-resolved photoelectron spectrum (TRPES) for $\mathrm{NH}_{3}$ evolving in the $B^{1} E^{\prime \prime}$ state. We label the vibrational states of $B^{1} E^{\prime \prime}$ as $\left(v_{1} v_{2} v_{3} v_{4}\right), v_{1}$ representing the 


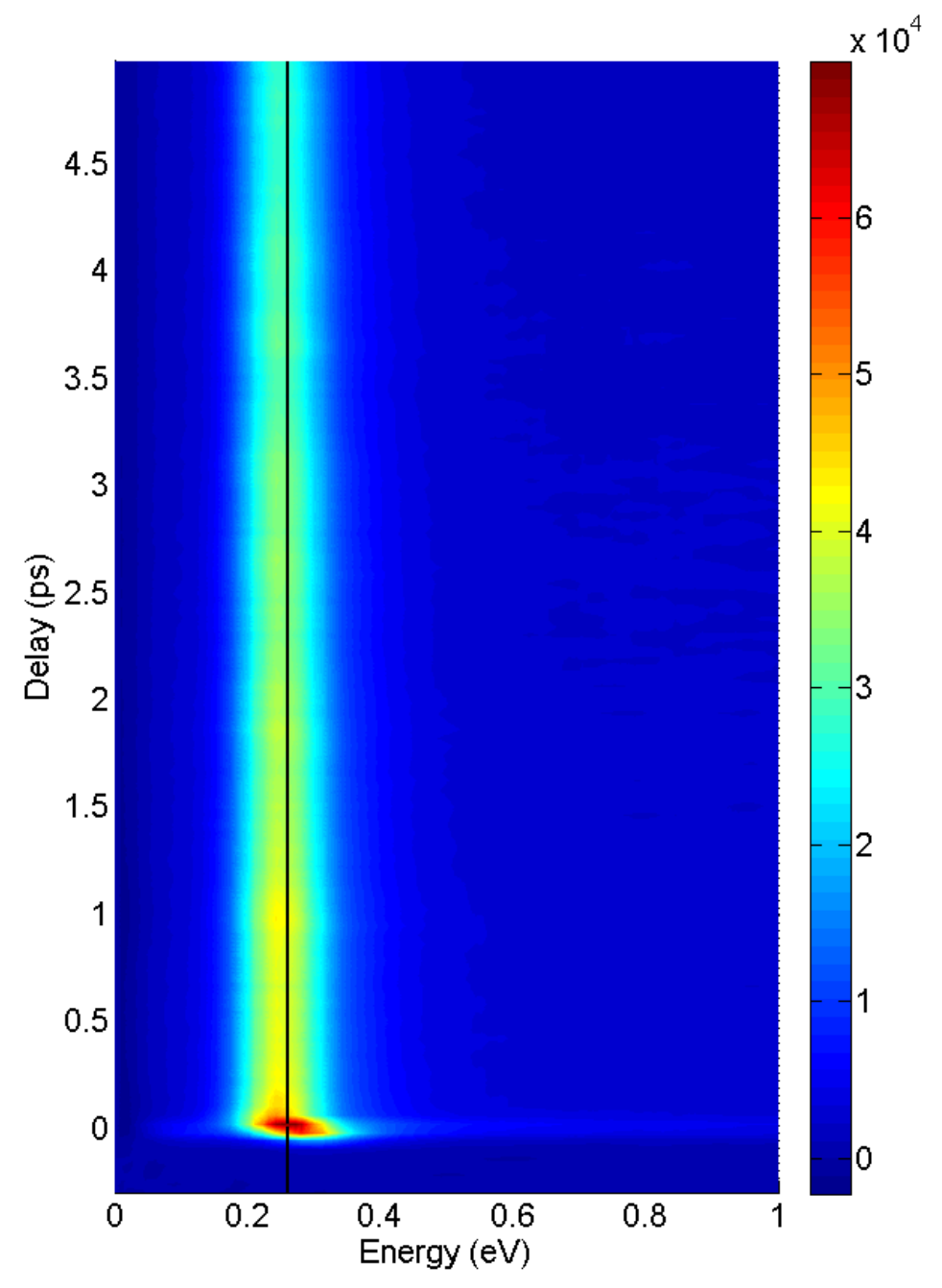

FIG. 2. Experimental TRPES spectrum for $\mathrm{NH}_{3}$ pumped at $160.9 \mathrm{~nm}$ and probed at 400nm. The color scale represents the photoelectron yield in arbitrary units. The vertical solid line marks the expected photoelectron energy for ionization into the (0300) $X^{2} A_{2}^{\prime \prime}$ cationic state.

number of quanta in the symmetric stretch mode, $v_{2}$ in the symmetric umbrella mode, $v_{3}$ in the asymmetric stretch mode and $v_{4}$ in the asymmetric bend mode. The $62,150 \mathrm{~cm}^{-1}$ pump pulse excites the (0300) umbrella mode state. The peak of the photoelectron spectrum at $0.26 \mathrm{eV}$ is consistent with ionization into the (0300) $X^{2} A_{2}^{\prime \prime}$ ground ionic state, as expected since the $B^{1} E^{\prime \prime}$ state is a member of the Rydberg series converging to this state [47]. The expected photoelectron energy for ionization into this state is marked by the vertical, black solid line in the figure. This is as expected for direct photoionization provided the pumped 
and ionic states have parallel potential energy surfaces, implying an essentially diagonal Franck-Condon matrix. This strongly indicates that resonant autoionization is negligible in our experiment.

\section{B. Theoretical Model}

Here we describe the model used to calculate the time dependent ADMs and EADMs constituting the LF electronic charge distribution. The doubly degenerate $\tilde{B}^{1} E^{\prime \prime}$ electronic state of $\mathrm{NH}_{3}$ was studied in detail using a variety of frequency domain spectroscopies [4750]. The one-photon absorption spectrum exhibits a series of bands assigned to sequentially increasing quanta of the umbrella mode. These result from a change in geometry from the pyramidal $C_{3 v}$ ground state to the planar $D_{3 h}$ equilibrium geometry of the degenerate $\tilde{B}^{1} E^{\prime \prime}$. The Jahn-Teller theorem necessitates a distortion of this geometry via the asymmetric vibrational modes. This results in a series of umbrella mode - asymmetric stretch combination lines also being observed in the absorption spectrum. However, in this experiment the nondegenerate vibrational state excited by the pump pulse - (0300) - is not Jahn-Teller active (i.e. no asymmetric mode excitation). In (0300), the degenerate electronic components are split by coupling of the rotational and electronic angular momenta as a result of the Coriolis effect, L-uncoupling and the Jahn-Teller distortion of the state [49, 51]. The rotational states excited by the pump pulse are described by the following Hamiltonian which accounts for these effects $[49,51]$

$$
\begin{aligned}
H= & T_{v}+B J_{\Lambda}\left(J_{\Lambda}+1\right)+(C-B) K_{\Lambda}^{2}-D_{J_{\Lambda}} J_{\Lambda}^{2}\left(J_{\Lambda}+1\right)^{2}-D_{J_{\Lambda} K_{\Lambda}} J_{\Lambda}\left(J_{\Lambda}+1\right) K_{\Lambda}^{2}-D_{K_{\Lambda}} K_{\Lambda}^{4} \\
& -2 C \zeta K_{\Lambda} \Lambda+q / 2\left(L_{+}^{2} J_{-}^{2}-L_{-}^{2} J_{+}^{2}\right) .
\end{aligned}
$$

Here, $T_{v}$ is the term energy for a particular vibronic state and $B$ and $C$ are the symmetric top rotational constants in that state. $\Lambda= \pm 1$ label the degenerate electronic components (corresponding to our electronic indices $n$ and $n^{\prime}$ above) of the $\tilde{B}^{1} E^{\prime \prime}$ state, with $J_{\Lambda}$ and $K_{\Lambda}$ being the corresponding total angular momentum (neglecting spin) and its projection onto the body axis of highest symmetry (the $C_{3}$ axis for $\mathrm{NH}_{3}$ ). $D_{J_{\Lambda}}, D_{J_{\Lambda} K_{\Lambda}}$ and $D_{K_{\Lambda}}$ are coefficients that quantify centrifugal distortion in a particular $J_{\Lambda}, K_{\Lambda}$ state. The first line in the above equation, containing only these parameters and operators, constitutes the standard 
Hamiltonian for a symmetric top including centrifugal distortion. In the next line, $\zeta$ is the Coriolis coupling constant, containing matrix elements of the electronic angular momentum operator coupling the degenerate electronic states, to nearby states of appropriate symmetry. Specifically, the $\Lambda=+1$ component interacts strongly with the nearby $\tilde{C}^{\prime 1} A_{1}^{\prime}$ state, resulting in splitting of the $\Lambda= \pm 1$ components. This is exactly analogous to $\Lambda$ doubling in $\Pi$ states of diatomic molecules [21]. The parameter $q$ similarly contains matrix elements coupling these electronic states, as well a nuclear mass dependent term that is a result of Jahn-Teller distortion in the electronic state [51]. The operators $J_{ \pm}$and $L_{\mp}$ are raising and lowering operators of the total projection angular momentum $K$, and electronic states $\Lambda$, respectively. The $q$ containing term mixes $\Lambda= \pm 1$ states, with $K_{\lambda}$ separation $\left|K_{1}-K_{-1}=2\right|$. In the separable rotational-electronic basis $\left|J_{\Lambda} K_{\Lambda} M_{\Lambda}\right\rangle|\Lambda\rangle$, the Hamiltonian matrix is,

$$
\begin{aligned}
& H_{J_{\Lambda} K_{\Lambda} \Lambda, J_{\Lambda^{\prime}} K_{\Lambda^{\prime}}^{\prime}}=\delta_{J_{\Lambda} J_{\Lambda^{\prime}}^{\prime}, K_{\Lambda} K_{\Lambda^{\prime}}^{\prime}, \Lambda \Lambda^{\prime}} E_{0} \\
& +\delta_{\Lambda^{\prime} \Lambda \pm 2, K_{\Lambda^{\prime}}^{\prime} K_{\Lambda} \pm 2} \frac{q}{2} \sqrt{\left(J_{\Lambda}\left(J_{\Lambda}+1\right)-K_{\Lambda}\left(K_{\Lambda}+1\right)\right)\left(J_{\Lambda}\left(J_{\Lambda}+1\right)-K_{\Lambda^{\prime}}^{\prime}\left(K_{\Lambda^{\prime}}^{\prime} \pm 1\right)\right)}, \\
& E_{0}=T_{v}+B J_{\Lambda}\left(J_{\Lambda}+1\right)+(C-B) K_{\Lambda}^{2} \\
& -D_{J_{\Lambda}} J_{\Lambda}^{2}\left(J_{\Lambda}+1\right)^{2}-D_{J_{\Lambda} K_{\Lambda}} J_{\Lambda}\left(J_{\Lambda}+1\right) K_{\Lambda}^{2}-D_{K_{\Lambda}} K_{\Lambda}^{4}-2 C \zeta K_{\Lambda} \Lambda .
\end{aligned}
$$

The Hamiltonian couples zeroth order states with $\Delta K_{\Lambda, \Lambda^{\prime}}=\Delta \Lambda=2$, as a result of the loss of three-fold rotational symmetry. The parameters in this Hamiltonian that provide spectroscopically accurate rotational energies for the (0300) state were determined by Ashfold et al. [49], and are given here in Table I. The eigenstates of this Hamiltonian can be expressed as,

$$
\psi_{J M \gamma}=v_{K_{\Lambda}, \Lambda}^{(J M \gamma)}\left|J_{\Lambda} K_{\Lambda} M_{\Lambda} \Lambda\right\rangle+v_{K_{\Lambda \pm 2} \pm 2, \Lambda \pm 2}^{(J M \gamma)}\left|J_{\Lambda \pm 2} K_{\Lambda \pm 2} \pm 2 M \Lambda \pm 2\right\rangle,
$$

where eigenstates are labeled by $\gamma$ in order of increasing energy. The $v_{K_{\Lambda}, \Lambda}^{(J M \gamma)}$ are coefficients of the eigenvector $\gamma$ of the Hamiltonian matrix (Eq. 5) for a particular $J, M$ state. Fig. 3 shows the resulting rotational level structure.

The wavepacket excited by the pump pulse can then be expressed as a coherent superposition of these eigenstates,

$$
\Psi(t)=\sum_{J M \gamma} a_{J M \gamma} \psi_{J M \gamma} e^{2 \pi i E_{J M \gamma} / t},
$$

where $E_{J M \gamma}$ are the eigenvalues of Eq. 5. The amplitude coefficient $a_{J M \gamma}$ is proportional to the dipole transition amplitude into the eigenstate $\psi_{J M \gamma}$. This in turn is proportional to a 


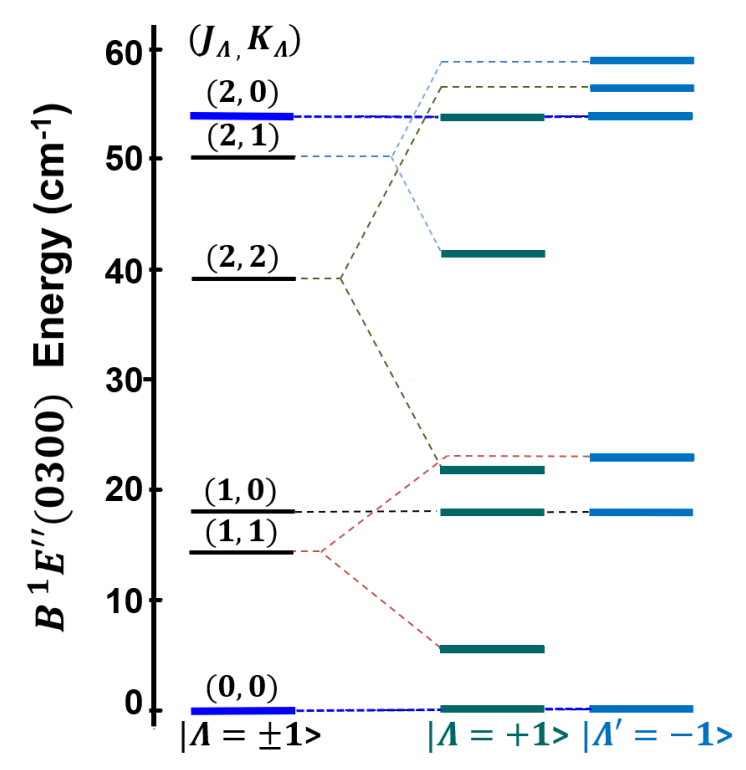

FIG. 3. Level structure of the $\tilde{B}^{1} E^{\prime \prime}$ state of $\mathrm{NH}_{3}$. On the left (black) are levels of the uncoupled degenerate electronic state $|\Lambda= \pm 1\rangle$. For $K_{\Lambda}=0$ states, the Coriolis coupling vanishes and these remain eigenstates. On the right, when $K_{\Lambda}>0$, the linear Coriolis interaction lifts the degeneracy, yielding two electronic states labeled $|\Lambda=+1\rangle$ (moss green) and $\left|\Lambda^{\prime}=-1\right\rangle$ (steel blue), each with their level structure. The quadratic Coriolis interaction, as well the Jahn-Teller effect further mixes the states to give the eigenstates in eq. 6. The shift in energy due to this Jahn-Teller mixing is small on the scale shown here, but increases with $K$.

\begin{tabular}{|c|c|}
\hline $\mathrm{T}_{v}$ & $62015.232 \mathrm{~cm}^{-1}$ \\
\hline $\mathrm{B}$ & $8.9679 \mathrm{~cm}^{-1}$ \\
\hline $\mathrm{C}$ & $5.2964 \mathrm{~cm}^{-1}$ \\
\hline $\mathrm{D}_{J_{ \pm 1}}$ & $-520 \mathrm{e}-6 \mathrm{~cm}^{-1}$ \\
\hline $\mathrm{D}_{J_{ \pm 1} K_{ \pm 1}}$ & $1963 \mathrm{e}-6 \mathrm{~cm}^{-1}$ \\
\hline $\mathrm{D}_{K_{ \pm 1}}$ & $-1801 \mathrm{e}-6 \mathrm{~cm}^{-1}$ \\
\hline$\zeta$ & $0.8177 \mathrm{~cm}^{-1}$ \\
\hline $\mathrm{q}$ & $0.5399 \mathrm{~cm}^{-1}$ \\
\hline
\end{tabular}

TABLE I. Parameters for the effective Hamiltonian in Eq. 4 
superposition of rotational transition amplitudes between the ground and excited state,

$$
\begin{array}{r}
a_{J M \gamma}=v_{K_{-1},-1}^{(J M \gamma)} g\left(J_{0}^{\prime \prime}, K_{0}^{\prime \prime}, J_{\Lambda}, K_{\Lambda}, M_{\Lambda}, 1,-1\right)+v_{K_{1}, 1}^{(J M \gamma)} g\left(J_{0}^{\prime \prime}, K_{0}^{\prime \prime}, J_{\Lambda}, K_{\Lambda}, M_{\Lambda}, 1,1\right), \\
g\left(J_{0}^{\prime \prime}, K_{0}^{\prime \prime}, J_{\Lambda}, K_{\Lambda}, M_{\Lambda}, 1, q\right)=\sqrt{\frac{2 J^{\prime \prime}+1}{2 J+1}}\left\langle J_{0}^{\prime \prime} M_{0} ; 10 \mid J_{\Lambda} M_{\Lambda}\right\rangle\left\langle J_{0}^{\prime \prime} K_{0}^{\prime \prime} ; 1 q \mid J_{\Lambda} K_{\Lambda}\right\rangle .
\end{array}
$$

The factors $g\left(J_{0}^{\prime \prime}, K_{0}^{\prime \prime}, J_{\Lambda}, K_{\Lambda}, M_{\Lambda}, 1, q\right)$ are rotational transition amplitudes between the ground, symmetric top state $\left|J_{0}^{\prime \prime} K_{0}^{\prime \prime} M_{0}\right\rangle$ and the symmetric top basis state in the excited electronic manifold $\left|J_{\Lambda} K_{\Lambda} M_{\Lambda}\right\rangle$, via the spherical tensor dipole transition operator $\mu_{q}^{1}$. It is additionally assumed that the electronic dipole matrix elements are related by the expression $\left\langle 1\left|\mu_{1}^{1}\right| 0\right\rangle=\left\langle-1\left|\mu_{-1}^{1}\right| 0\right\rangle$, where $|0\rangle$ is the ground state electronic wavefunction. These matrix elements, along with a transition energy dependent perturbative weighting factor constitute the overall proportionality constant, which is neglected here. The transition energy dependence here can be neglected due to the relatively small spacing between rotational states. The Clebsch-Gordan coefficients $\langle. . ; . . \mid .$.$\rangle in above expression enforce the conservation$ of angular momentum by the selection rules $\Delta J_{0, \Lambda}=0, \pm 1, \Delta M_{0, \Lambda}=0$ for the rotational eigenstates, and $\Delta K_{0, \Lambda}= \pm 1, \Delta \Lambda=\Delta K_{0, \Lambda}$ for the symmetric top and electronic basis states.

The ground state distribution of rotational states is simulated by a Boltzmann distribution of symmetric top states, with rotational constants $A=B=9.44430 \mathrm{~cm}^{-1}$ and $C=6.19600 \mathrm{~cm}^{-1}$. An additional weight is included which depends on the symmetry of the nuclear spin wavefunction that must be grouped with the correct rotational wavefunction such that the Pauli Exclusion Principle is obeyed. Rotational states with $K_{0}=3 m$, where $m$ is an integer, must be grouped with para spin states and those with $K_{0} \neq 3 m$ with ortho spin states. The 2: 1 ratio of ortho to para spin states is used as a weight for the corresponding rotational states. For $K_{0}=0$, only $A_{2}^{\prime}$ or $A_{2}^{\prime \prime}$ symmetry representations in $D_{3 h}$ can be paired with ortho or para states such that Pauli exclusion is obeyed. The final state (0300) has $E^{\prime}$ symmetry, rendering only transitions out of $A_{2}^{\prime \prime}$ states as dipole allowed. Since only odd $J$ states in the ground electronic state can generate $A_{2}^{\prime \prime}$ symmetry, this means that transitions out of even $J$ states with $K=0$ are forbidden and therefore excluded here. A complete description of these symmetry selection rules is provided in previous spectroscopic studies $[47]$.

To calculate the ADMs and EADMs (Eq. 3), we require the density matrix in the $\left|J_{\Lambda} K_{\Lambda} M_{\Lambda} \Lambda\right\rangle$ basis. Writing $A_{J M \gamma}(t)=a_{J M \gamma} e^{2 \pi i E_{J M \gamma} / t}$, the amplitude coefficients $c_{J_{\Lambda} K_{\Lambda} M_{\Lambda}}^{(\Lambda)}(t)$ 
in the $|J K M \lambda\rangle$ basis are calculated using the eigenvectors $v_{K_{\Lambda}, \Lambda}^{(J M \gamma)}$,

$$
c_{J_{\Lambda} K_{\Lambda} M_{\Lambda}}^{(\Lambda)}(t)=\sum_{\gamma} v_{K, \Lambda}^{(J M \gamma)} A_{J M \gamma}(t)
$$

The density matrix is then straight-forwardly given by $\rho_{\omega_{\Lambda}, \omega_{\Lambda^{\prime}}^{\prime}}\left(\Lambda, \Lambda^{\prime}\right)(t)=$ $c_{J_{\Lambda} K_{\Lambda} M_{\Lambda}}^{(\Lambda)}(t) c_{J_{\Lambda^{\prime}}^{\prime} K_{\Lambda^{\prime}}^{\prime} M_{\Lambda^{\prime}}^{\prime}}^{\left(\Lambda^{\prime}\right) *}(t)$, where $\omega_{\Lambda}$ refers collectively to the angular momentum quantum numbers associated with the electronic state $\Lambda$. Wavepackets initialized from each initial rotational state in the ground state manifold are propagated independently, and the ADMs and EADMs calculated by replacing $n$ with $\Lambda$ in Eq. 3. Each of these are then ascribed a thermal weight depending on the initial state, calculated as described above. The weighted ADMs and EAMDs are then summed to provide the final, thermally averaged result presented in the next section. Note in addition that the selection rules out of the ground state given above only allow the angular momentum coherences $\left|J_{\Lambda}-J_{\Lambda^{\prime}}^{\prime}\right|=2,\left|K_{\Lambda}-K_{\Lambda^{\prime}}^{\prime}\right|=0$ and $\left|J_{\Lambda}-J_{\Lambda^{\prime}}^{\prime}\right|=2,\left|K_{\Lambda}-K_{\Lambda^{\prime}}^{\prime}\right|=2$ to be excited. Therefore, the only ADMs that need to be considered are $A_{00}^{2}( \pm 1, \pm 1 ; t)$ and $A_{0 \pm 2}^{2}( \pm 1, \mp 1 ; t)$.

\section{Results and Discussion}

In our demonstration, the pump laser prepared only a single vibrational state (0300) within each electronic state, producing a ro-electronic wavepacket having constant vibrational quantum number. In Fig.1(a), this corresponds to the situation where both $\Delta E_{\text {elec }}$ and the laser bandwidth are smaller than the vibrational level spacings. The two coherently prepared electronic states $|n\rangle$ and $\left|n^{\prime}\right\rangle$ in our wavepacket are the states $|\Lambda\rangle$, where $\Lambda= \pm 1$. Our pump pulse prepared a ro-electronic wavepacket with both intrastate and interstate coherences. The intrastate coherences (blue arrows) are quantified by the ADMs, the interstate electronic coherences (red arrows) by the EADMs. In Fig. 1(c), we show a calculated MF electronic density, at three time delays, for the specific EADM coherence represented by the upper red arrow in Fig. 1(a). Details of this calculation are provided in an appendix. In $\mathrm{NH}_{3} \tilde{B}^{1} E^{\prime \prime}$, due to the small electronic bandgap, the electronic dynamics are on times scale similar but demonstrably not identical to that of rotation. We emphasize that this demonstration in no way negates the generality of our approach. The highest moment of the TRPAD will always separate electronic coherences from populations and, for the general case of valence electronic wavepackets, further separate rotational from electronic coherences 


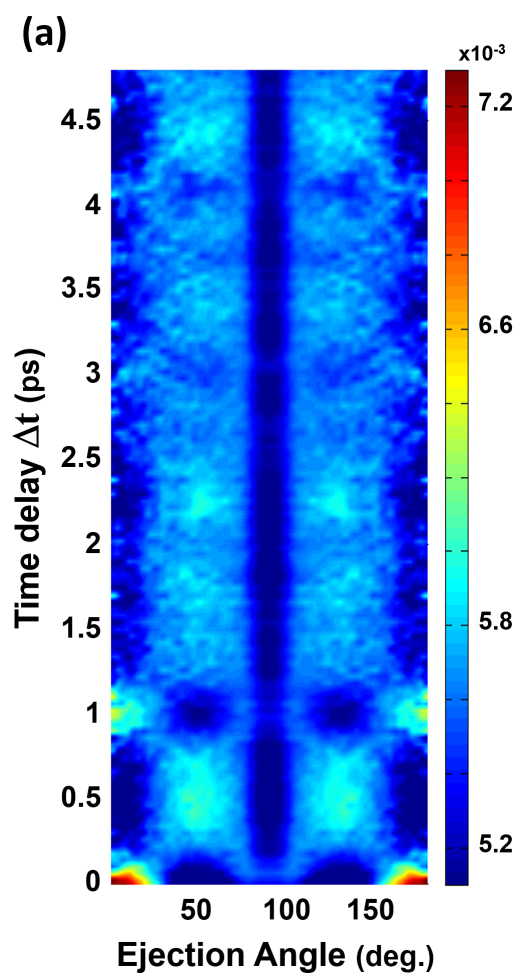

(b)
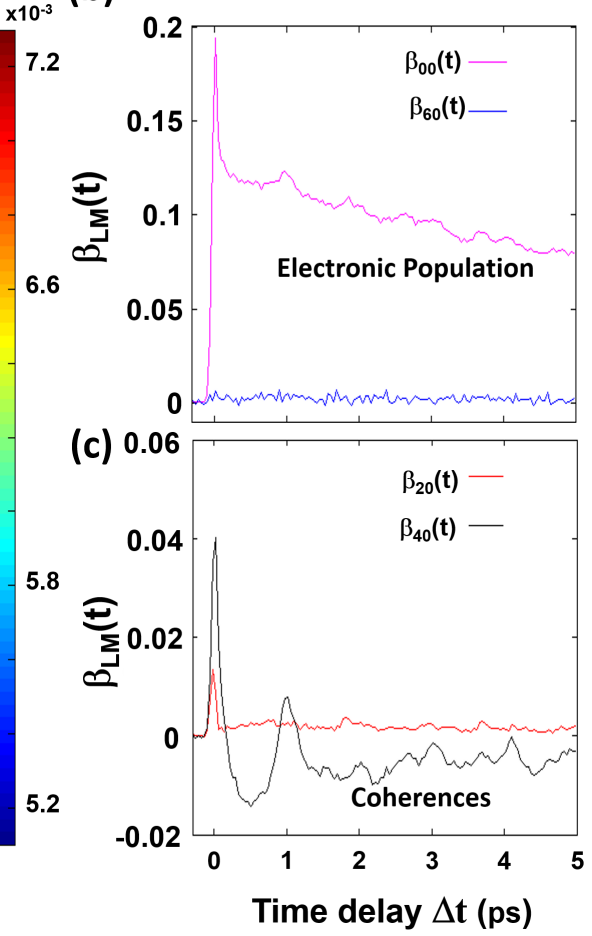

FIG. 4. (a) The experimental TRPAD for the observed photoelectron band from an evolving wavepacket in the degenerate $\tilde{B}^{1} E^{\prime \prime}$ electronic state of $\mathrm{NH}_{3}$. The angle (degrees) on the x-axis is the polar ejection angle $\theta_{e}$, plotted as a function of time delay (y-axis). (b)-(c) The $\beta_{L M}$ parameters extracted from fits to the data in (a) (see text).

based on time scales. It is interesting to compare with 2D electronic spectroscopies which also separate coherences from populations $[7,18,52,53]$ : these are based on 2D probing of MF coherences. Our method is a general LF pump-probe approach that applies to any system for which total angular momentum is conserved.

In Fig. 4, the experimental time-, energy- and angle-resolved TRPAD shows large variations. In general, the TRPADs can be phenomenologically fit to a moment expansion, $S\left(t, \epsilon, \theta_{e}, \phi_{e}\right)=\sum_{L M} \beta_{L M}(t, \epsilon) Y_{L M}\left(\theta_{e}, \phi_{e}\right)$, where $t$ is the time delay, $\epsilon$ the kinetic energy and $\theta_{e}, \phi_{e}$ the polar and azimuthal photoelectron ejection angles. This sum truncates by

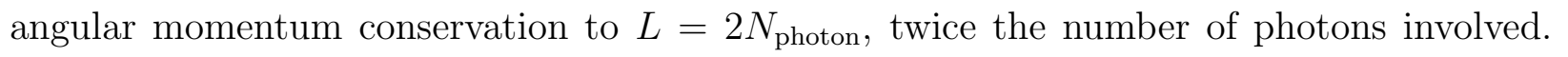
For a collinearly polarized one-photon pump, one-photon probe $\left(1+1^{\prime}\right)$ process, the sum truncates at $L=4$, with $M=0$. All experimental information on the excited state dynamics is contained in the $\beta_{L M}(t, \epsilon)$. Since in this particular case only a single photoelectron energy 
band is observed, we suppress in the following the dependence on kinetic energy. In Fig. 2 (b)-(c), we plot the fitted $\beta_{L M}(t)$ : it can be seen that the largest variations were observed in $\beta_{40}(t)$, Fig. 2(c). By comparison, $\beta_{20}(t)$ shows only small variations. $\beta_{00}(t)$, in Fig. $2(\mathrm{~b})$, is the evolving total ionization cross section (photoelectron yield, also shown in the SM) and shows population dynamics due to the well known predissociation via the $\tilde{A}^{1} A_{2}^{\prime \prime}$ state into $\mathrm{NH}_{2}+\mathrm{H}[54,55]$. We note that $\beta_{60}(t)$ should vanish by angular momentum conservation for $\left(1+1^{\prime}\right)$ processes, as shown here in Fig. 2(b): this confirms the systematic and statistical accuracy of our fits. These $\beta_{L 0}(t)$ contain distinctive contributions from the ADMs, the EADMs and the electronic population dynamics. As detailed below, $\beta_{40}(t)$ completely separates the electronic coherences from population dynamics.

We develop the relationship between the LF scattering anisotropies in Fig. 2 and the MF angular momentum coherences in Fig. 1(a). The $\beta_{L M}(t)$ can be expressed in terms of ADMs and EADMs as [23, 25]

$$
\begin{aligned}
\beta_{L M}(t, \epsilon) & =\sum_{P K Q S} \sum_{n} C_{P K Q S}^{L M}(n, n ; \epsilon) A_{Q S}^{K}(n, n ; t) \\
& +\sum_{P K Q S} \sum_{n \neq n^{\prime}} C_{P K Q S}^{L M}\left(n, n^{\prime} ; \epsilon\right) A_{Q S}^{K}\left(n, n^{\prime} ; t\right) .
\end{aligned}
$$

We separated this equation into two sums, the first reflecting intrastate rotational dynamics $(\mathrm{ADMs})$, the second interstate electronic coherences (EADMs). The coefficients $C_{P K Q S}^{L M}\left(n, n^{\prime} ; \epsilon\right)$ contain the energy-dependent photoionization dipole matrix elements, where $P=0,2$ for one-photon ionization with linearly polarized light. These coefficients link coherences to observables, decomposing the $\beta_{L M}(t, \epsilon)$ into ADMs and EADMs. Importantly, angular momentum selection rules limit the allowed values of $K, Q$ and $S[23,25,56]$. For linearly polarized one-photon excitation, only three possible ADMs/EADMs exist: $A_{00}^{0}\left(n, n^{\prime} ; t\right)$, $A_{00}^{2}\left(n, n^{\prime} ; t\right)$ and $A_{0 \pm 2}^{2}\left(n, n^{\prime} ; t\right)$. Linear superpositions of these contribute to the observed $\beta_{L M}(t, \epsilon)$, with angular momentum constraints permitting some separation. The sum of the ionizing photon angular momentum $P$ plus the ADM/EADM angular momentum $K$ must be partitioned between the angular momentum $L$ of the ejected photoelectron and that remaining in the ion. The electronic population dynamics always contribute to $\beta_{00}(t, \epsilon)$, may contribute to $\beta_{20}(t, \epsilon)$, but never to $\beta_{40}(t, \epsilon)$. Crucially, this permits a complete separation of electronic population dynamics from coherences: $\beta_{40}(t, \epsilon)$ is sensitive only to coherences [27] between states of differing total angular momentum, $J_{n}$. For a $\left(1+1^{\prime}\right)$ TRPAD experi- 

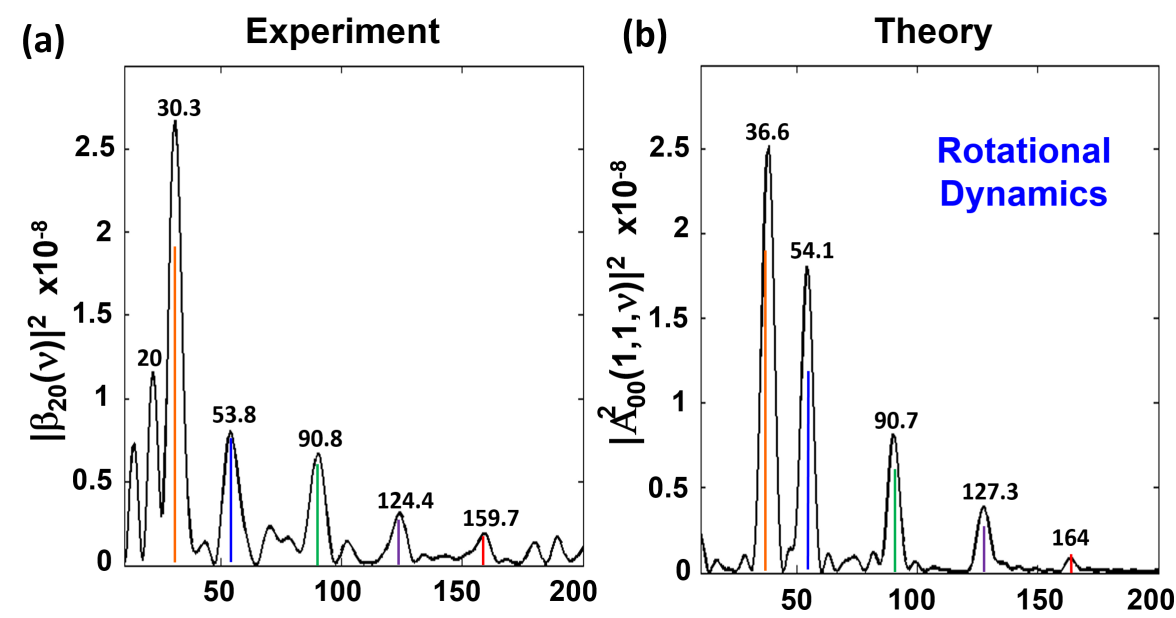

(c)

(d)
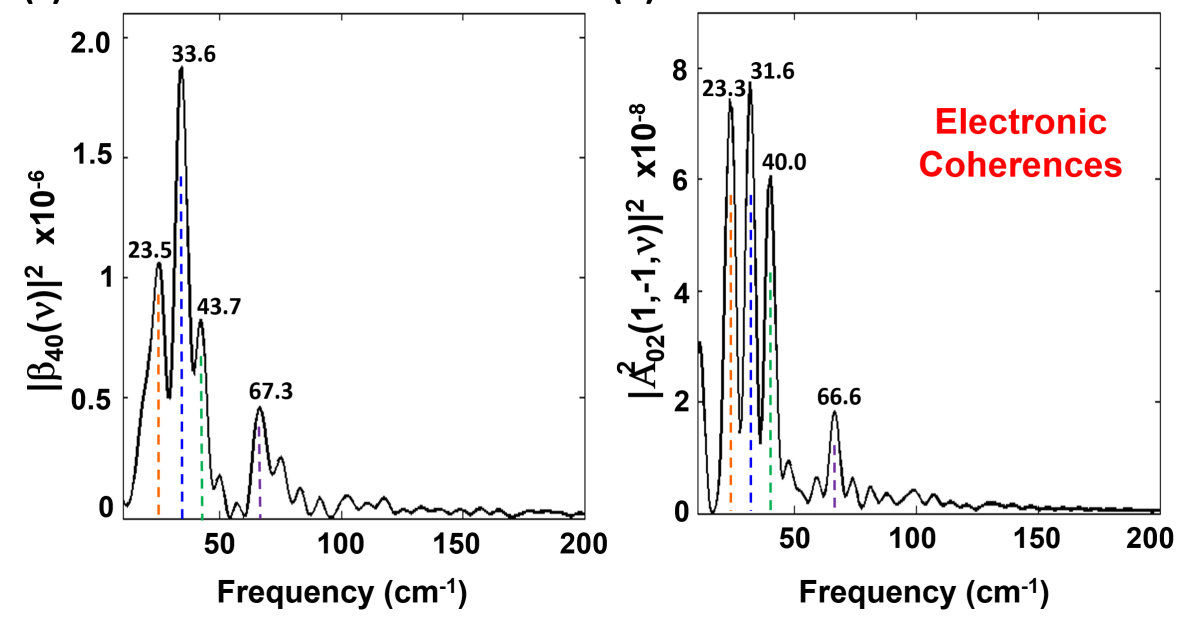

FIG. 5. Fourier power spectra of the time dependent data from Fig. 2(c) and their comparison with theory. (a) $\left|\beta_{20}(\nu)\right|^{2}$ from $\beta_{20}(t)$; (b) $\left|A_{00}^{2}(1,1 ; \nu)\right|^{2}$ from the calculated $A_{00}^{2}(1,1 ; t) ;(\mathrm{c})\left|\beta_{40}(\nu)\right|^{2}$ from $\beta_{40}(t)$; and (d) $\left|A_{02}^{2}(1,-1 ; \nu)\right|^{2}$ from the calculated $A_{02}^{2}(1,-1 ; t)$. As discussed in the SM, the counterpart of $(\mathrm{b}),\left|A_{00}^{2}(-1,-1 ; \nu)\right|^{2}$, tracking the rotational dynamics in the electronic state $\Lambda=-1$, is not shown since it is identical to (b). Similarly, the counterpart of $(\mathrm{d}),\left|A_{0-2}^{2}(-1,1 ; \nu)\right|^{2}$, contains no new frequency components and is not shown.

ment, $L=4$ results from $K=2$ and $P=2$, restricting the sum over $K Q S$ in Eq. 10 to only terms with $K=2$. Therefore, $K=0$ functions $A_{00}^{0}\left(n, n^{\prime} ; t\right)$, containing the electronic population dynamics for $n=n^{\prime}$, cannot possibly contribute. Hence, $\beta_{40}(t, \epsilon)$ is selectively sensitive to intrastate and interstate angular momentum coherences, but never to the population dynamics. In Fig. 3, we show Fourier power spectra of $\beta_{20}(t)$ and $\beta_{40}(t)$ from Fig.2. $\left|\beta_{20}(\nu)\right|^{2}$ shows peaks assignable to symmetric top rotational coherences [22] with separa- 
tion $4 B, B$ being the rotational constant. $\left|\beta_{40}(\nu)\right|^{2}$, however, contains new frequencies. Since $\left|\beta_{40}(\nu)\right|^{2}$ is sensitive only to rotational or electronic angular momentum coherences, the new frequencies in $\left|\beta_{40}(\nu)\right|^{2}$ must therefore be electronic coherences. To confirm this, we computed the $\mathrm{ADM} A_{00}^{2}(1,1 ; t)$ and $\operatorname{EADM} A_{02}^{2}(1,-1 ; t)$ (see SM for details). The good agreement between calculated and observed frequencies confirms that $\beta_{20}(t)$ predominantly tracks rotational dynamics, whereas $\beta_{40}(t)$ predominantly tracks electronic coherences. The TRPAD in Fig. 4(a), dominated by $\beta_{40}(t)$, is a direct experimental map of the time varying LF electronic density. The latter in turn reflects the electronic coherences, the MF charge oscillations resulting from non-adiabatic Coriolis dynamics (Fig. 1 (c)).

The strong separation of ADMs from EADMs in $\beta_{40}(t)$ observed here is unlikely to be accidental and the underlying reason for this is contained in the variation of the MF electronic wavefunction between the two eigenstates involved in a quantum beat. For the observed photoelectron angular distribution (PAD) to vary with time delay in a given quantum beat (the coherent sum of which is the wavepacket signal), the set of partial waves produced by each ionizing transition must differ in amplitude, phase or both: if they were identical, only the photoelectron yield and not the shape of the PAD would vary with time delay. The fact that that the shape of the PAD demonstrably varies with delay is proof that the set of partial waves produced by ionizing the two eigenstates involved in the quantum beat must be different. In particular, if $\beta_{40}(t)$ varies more strongly than $\beta_{20}(t)$, this difference must be greater for the higher $l$ than the lower $l$ partial wave components.

The well-known Wigner-Eckart theorem determines that the photoionization matrix element can, in complete generality, be separated into a geometrical factor and a dynamical factor (the reduced matrix element - the vibronic part). For the intrastate ionization transitions (the ADMs), the reduced matrix element should remain essentially the same since, by definition, they originate from the same electronic and (here) vibrational state: the only variation between eigenstates is therefore due to the geometrical factor alone. In contrast, for the interstate ionization transitions (the EADMs), both the geometrical factor and the reduced vibronic matrix element will vary, since by definition they originate from different electronic states. Therefore, one might expect that the difference in partial wave distributions (between the two eigenstates in the quantum beat) is more likely to be larger for interstate rather the intrastate coherences. The extent to which this larger difference appears in the higher $l$ or lower $l$ partial wave components will likely depend on the specific 
differences between shapes of the electronic wavefunctions involved. For the case of $\mathrm{NH}_{3}$, even though their time scales are (unfavourably) similar, the separation of rotational from electronic coherences is still demonstrably excellent.

We again state our second key point: $\beta_{40}(t)$, the highest moment of the scattering anisotropy (containing the EADMs), completely separates electronic coherences from population dynamics (which dominate the $\beta_{00}(t)$ signal). This result emerges from our analysis of the TRPADs in terms of ADMs and EADMs, the latter being introduced here for this purpose.

\section{SUMMARY AND OUTLOOK}

We presented a wavepacket method, deriving from angular momentum correlations, for studying electronic coherences in isolated systems which (i) completely separates electronic population dynamics from electronic coherences and (ii) is based on ultrafast angle-resolved scattering measurements. We introduced the EADM, a measure of interstate electronic angular momentum coherence which is distinct from intrastate rotational coherences (ADMs). The highest moment of our observed LF scattering anisotropy, $\beta_{40}(t)$, completely separates electronic populations from coherences, a long standing goal. To a high degree, it further separates rotational from electronic coherences. The EADMs generate an LF electronic anisotropy observable in principle using any ultrafast angle-resolved scattering probe, be it electron or X-ray scattering or, as shown here, angle-resolved photoelectron spectroscopy (TRPADs). In general, EADMs for molecular valence states will be driven by electronic coherences on attosecond to few-fs time scales, determined by electronic energy gaps. Electronic coherences in isolated molecules driven by vibrational dynamics near conical intersections [7], will generate short-lived (on the order of inverse vibrational level spacings) LF electronic anisotropies observable by lab-based attosecond angle-resolved photoemission $[57,58]$, or by time-resolved X-ray scattering such as becoming available at X-ray Free Electron Lasers (XFELs) [59]. With these tools in hand, the exploration of electronic coherences in molecular systems [60] will surely accelerate. 


\section{APPENDIX - WAVEFUNCTION REPRESENTATION AND ELECTRONIC DENSITY CALCULATIONS}

The time varying electronic probability densities shown in Fig. 1(c) were calculated by recasting Eq. 6 in the $|J K M \Lambda\rangle$ basis, using Eq. 9. For excitation out of a fixed initial state, Eq. 6 becomes

$$
\Psi(t)=\sum_{J_{\Lambda} K_{\Lambda} M_{\Lambda} \Lambda} c_{J_{\Lambda} K_{\Lambda} M_{\Lambda}}^{(\Lambda)}(t)\left|J_{\Lambda} K_{\Lambda} M_{\Lambda} \Lambda\right\rangle
$$

where the basis function amplitudes can be calculated using Eq. 9 and the procedure described in Section III B. Given the $c_{J_{\Lambda} K_{\Lambda} M_{\Lambda}}^{(\Lambda)}(t)$, the wavepacket can be projected onto eigenfunctions of the electronic coordinate space $\left\{\boldsymbol{r}_{i} \mid i=1, \ldots, N\right\}$ and the Euler angles $\Omega=\{\phi, \theta, \chi\}$ to get the time varying probability density. Projecting the basis onto the Euler angles gives the symmetric top basis functions,

$$
\left\langle\Omega \mid J_{\Lambda} K_{\Lambda} M_{\Lambda} \Lambda\right\rangle=\sqrt{\frac{2 J+1}{8 \pi^{2}}} D_{M_{\Lambda} K_{\Lambda}}^{J_{\Lambda}^{*}}(\Omega)|\Lambda\rangle .
$$

Defining the projection of the electronic basis functions onto the basis of electronic coordinates as $\left|\Lambda\left(\boldsymbol{r}_{1}, \ldots, \boldsymbol{r}_{N}\right)\right\rangle$, the total wavefunction can be written as,

$$
\psi\left(\Omega,\left\{\boldsymbol{r}_{i} \mid i=1, \ldots, N\right\}, t\right)=\Phi_{+1}(\Omega, t)\left|+1\left(\boldsymbol{r}_{1}, \ldots, \boldsymbol{r}_{N}\right)\right\rangle+\Phi_{-1}(\Omega, t)\left|-1\left(\boldsymbol{r}_{1}, \ldots, \boldsymbol{r}_{N}\right)\right\rangle
$$

This is a superposition of the MF electronic states $| \pm 1\rangle$, with $\Phi_{\Lambda}(\Omega, t)=\sum_{J_{\Lambda} K_{\Lambda} M_{\Lambda}} c_{J_{\Lambda} K_{\Lambda} M_{\Lambda}}^{(\Lambda)}(t)$ $D_{M_{\Lambda} K_{\Lambda}}^{J_{\Lambda}^{*}}(\Omega)$ being the amplitude of each electronic state $|\Lambda\rangle$. These can be interpreted as a rotational wavepackets in each electronic state. The time evolution of the total probability density $\left|\psi\left(\Omega,\left\{\boldsymbol{r}_{i} \mid i=1, \ldots, N\right\}, t\right)\right|^{2}$ is thus determined by three terms. Two population terms, $\left|\Phi_{\Lambda}(\Omega, t)\right|^{2}$, one for each electronic state, which represent the time evolving axis distribution and total population of the states. These only contain intrastate, rotational quantum beats $\left|J_{1}-J_{1}^{\prime}\right|=0,1,2$ and/or $\left|K_{1}-K_{1}^{\prime}\right|=0,2$. The $\operatorname{ADMs} A_{Q S}^{K}(\Lambda, \Lambda ; t)$ are proportional to angular moments of these distributions. The remaining interference or coherence term $2 \operatorname{Re}\left\{\Phi_{+1}(\Omega, t) \Phi_{-1}^{*}(\Omega, t)\right\}$, contains interstate quantum beats $\left|J_{1}-J_{-1}^{\prime}\right|=0,1,2$ and/or $\left|K_{1}-K_{-1}^{\prime}\right|=0,2$. The alignment angle dependence of this MF electronic coherence, which varies with time, introduces an LF anisotropy of the electronic probability distribution quantified by Eq. 2. The EADMs are proportional to angular moments of this cross term. Note that typically the rotational and electronic coordinates are uncoupled, and the electronic states are well separated. The axis distributions $\left|\Phi_{\Lambda}(\Omega, t)\right|^{2}$ thus typically 
evolve on a much slower time scale than the interference term $2 \operatorname{Re}\left\{\Phi_{+1}(\Omega, t) \Phi_{-1}^{*}(\Omega, t)\right\}$. The electronic LF anisotropy and the EADMs will therefore oscillate on this fast, electronic time scale (essentially a single quantum beat), unaffected by rotation over a relatively long time window, directly reflecting the MF electronic motion. However, in the specific case of $\mathrm{NH}_{3}\left(\tilde{B}^{1} E^{\prime \prime}\right)$ described here, the ADMs evolve on a time scale similar but not identical with that of the MF electronic motion. Therefore, since the electronic coherence term $2 \operatorname{Re}\left\{\Phi_{+1}(\Omega, t) \Phi_{-1}^{*}(\Omega, t)\right\}$ is alignment angle dependent, the evolving axis distribution in each state strongly influences the frequency and amplitude of the MF electronic motion, and correspondingly that of the electronic LF anisotropy and EADMs. Qualitatively, this can be thought of as the effect of the Coriolis force on the electron where the electron 'lags behind' the rotation of the molecular frame. Also note here that in the absence of the Coriolis and Jahn-Teller effects the electronic states $| \pm 1\rangle$ remain degenerate, rendering the electronic coherence term time-independent and the MF electronic charge distribution therefore appears stationary in the molecular frame.

Choosing $\left(J_{0}, K_{0}, M_{0}\right)=(1,1,0)$ as the initial rotational state in the ground state manifold, we calculated the $c_{J_{\Lambda} K_{\Lambda} M_{\Lambda}}^{(\Lambda)}(t)$. Selection rules discussed in Section III B only allow for excitation of $\left(J_{\Lambda}, K_{\Lambda}, M_{\Lambda}, \Lambda\right)=(2,2,0,1),(2,0,0,-1)$ and $(0,0,0,-1)$. The Wigner $D_{M_{\Lambda} K_{\Lambda}}^{J_{L^{*}}}(\Omega)$ matrix elements are tabulated analytical functions [56]. The electronic basis functions are calculated numerically as follows at the second-order algebraic diagramatic construction $(\mathrm{ADC}(2))$ level of theory using the $6-311++\mathrm{G}^{* *}$ basis set. Let $\gamma^{(0, \psi)}\left(\boldsymbol{r}_{E}, \boldsymbol{r}_{H}, \Omega, t\right)$ denote the one-electron transition density (1TDM) corresponding to excitation from the ground electronic state $|0\rangle$ to the state $|\psi\rangle$,

$$
\gamma^{(0, \psi)}\left(\boldsymbol{r}_{E}, \boldsymbol{r}_{H}, \Omega, t\right)=N \int_{-\infty}^{\infty} 0\left(\boldsymbol{r}_{H}, \boldsymbol{r}_{2}, \ldots, \boldsymbol{r}_{N}\right) \psi\left(\Omega, \boldsymbol{r}_{E}, \boldsymbol{r}_{2}, \ldots, \boldsymbol{r}_{N}, t\right) d \boldsymbol{r}_{2} \cdots d \boldsymbol{r}_{N}
$$

In the electron-hole picture, $\left|\gamma^{(0, \psi)}\left(\boldsymbol{r}_{E}, \boldsymbol{r}_{H}, \Omega, t\right)\right|^{2}$ represents the probability of finding the excited electron at $\boldsymbol{r}_{E}$ and the corresponding hole at $\boldsymbol{r}_{H}$, as a function of $\Omega$ and $t$. Integrating $\left|\gamma^{(0, \psi)}\left(\boldsymbol{r}_{E}, \boldsymbol{r}_{H}, \Omega, t\right)\right|^{2}$ over the hole coordinate $\boldsymbol{r}_{H}$ yields the angle- and time-dependent particle density $\rho\left(\Omega, \boldsymbol{r}_{E}, t\right)$,

$$
\rho\left(\Omega, \boldsymbol{r}_{E}, t\right)=\int_{-\infty}^{\infty}\left|\gamma^{(0, \psi)}\left(\boldsymbol{r}_{E}, \boldsymbol{r}_{H}\right)\right|^{2} d \boldsymbol{r}_{H}
$$

which can be interpreted as the probability of finding the excited electron at the position $\boldsymbol{r}_{E}$, as a function of $\Omega$ and $\mathrm{t}$. 
The practical calculation of the angle-dependent particle density makes use of the fact that the $1 \mathrm{TDM} \gamma^{(0, \psi)}\left(\boldsymbol{r}_{E}, \boldsymbol{r}_{H}, \Omega, t\right)$ can represented in the molecular orbital (MO) basis $\left\{\phi_{p}(\boldsymbol{r})\right\}$ as

$$
\gamma^{(0, \psi)}\left(\boldsymbol{r}_{E}, \boldsymbol{r}_{H}, \Omega, t\right)=\sum_{p q} d_{p q}^{(0, \psi)}(\Omega, t) \phi_{p}\left(\boldsymbol{r}_{E}\right) \phi_{q}\left(\boldsymbol{r}_{H}\right)
$$

Here, $\boldsymbol{d}^{(0, \psi)}(\Omega, t)$ denotes the one-electron transition density matrix between $|0\rangle$ and $|\psi(\Omega, t)\rangle$ :

$$
d_{p q}^{(0, \psi)}(\Omega, t)=\left\langle 0\left|\hat{a}_{p}^{\dagger} \hat{a}_{q}\right| \psi(\Omega, t)\right\rangle,
$$

where $\hat{a}_{p}^{\dagger}$ and $\hat{a}_{p}$ denote the elementary fermionic creation operators corresponding to the single-particle basis $\left\{\phi_{p}(\boldsymbol{r})\right\}$.

Fig. 1(c) shows the maximum value isosurfaces of $\rho\left(\Omega, \boldsymbol{r}_{E}, t\right)$ for $\Omega=(0, \pi / 2, \pi / 2)$ at time delays of $t=0,0.65$ and 1.05 ps.

\section{ACKNOWLEDGMENTS}

We thank D. Moffatt and D. Guay for technical support, and P. Hockett, M. Chergui, R.W. Field and J.G. Underwood for useful and interesting discussions. AS and MS thanks the NSERC Discovery Grant program for financial support. STP was supported by the GasPhase Chemical Physics Program within the U.S. Department of Energy, Office of Science, Office of Basic Energy Sciences, Division of Chemical Sciences, Geosciences, and Biosciences under contract No. DE-AC02-06CH11357.

[1] Yuki Kobayashi, Maurizio Reduzzi, Kristina F Chang, Henry Timmers, Daniel M Neumark, and Stephen R Leone. Selectivity of electronic coherence and attosecond ionization delays in strong-field double ionization. Physical Review Letters, 120(23):233201, 2018.

[2] F Calegari, D Ayuso, A Trabattoni, Louise Belshaw, Simone De Camillis, S Anumula, F Frassetto, L Poletto, A Palacios, P Decleva, et al. Ultrafast electron dynamics in phenylalanine initiated by attosecond pulses. Science, 346(6207):336-339, 2014.

[3] Peter M Kraus, Song Bin Zhang, A Gijsbertsen, RR Lucchese, N Rohringer, and Hans Jakob 
Wörner. High-harmonic probing of electronic coherence in dynamically aligned molecules. Physical Review Letters, 111(24):243005, 2013.

[4] Eleftherios Goulielmakis, Zhi-Heng Loh, Adrian Wirth, Robin Santra, Nina Rohringer, Vladislav S Yakovlev, Sergey Zherebtsov, Thomas Pfeifer, Abdallah M Azzeer, Matthias F Kling, et al. Real-time observation of valence electron motion. Nature, 466(7307):739, 2010.

[5] Artem Rudenko, Varun Makhija, Aram Vajdi, Thorsten Ergler, Markus Schürholz, Rajesh K Kushawaha, Joachim Ullrich, Robert Moshammer, and Vinod Kumarappan. Strong-fieldinduced wave packet dynamics in carbon dioxide molecule. Faraday Discussions, 194:463-478, 2016.

[6] David M Jonas. Vibrational and nonadiabatic coherence in 2D electronic spectroscopy, the Jahn-Teller effect, and energy transfer. Annual review of physical chemistry, 69:327-352, 2018.

[7] Markus Kowalewski, Kochise Bennett, Konstantin E Dorfman, and Shaul Mukamel. Catching conical intersections in the act: monitoring transient electronic coherences by attosecond stimulated X-ray Raman signals. Physical Review Letters, 115(19):193003, 2015.

[8] Markus Kowalewski, Kochise Bennett, and Shaul Mukamel. Monitoring nonadiabatic avoided crossing dynamics in molecules by ultrafast X-ray diffraction. Structural Dynamics, 4(5):054101, 2017.

[9] Caroline Arnold, Oriol Vendrell, Ralph Welsch, and Robin Santra. Control of nuclear dynamics through conical intersections and electronic coherences. Physical Review Letters, 120(12):123001, 2018.

[10] Mats Simmermacher, Niels E Henriksen, Klaus B Møller, Andrés Moreno Carrascosa, and Adam Kirrander. Electronic coherence in ultrafast X-ray scattering from molecular wave packets. Physical Review Letters, 122(7):073003, 2019.

[11] Yuki Kobayashi, Kristina F Chang, Tao Zeng, Daniel M Neumark, and Stephen R Leone. Direct mapping of curve-crossing dynamics in IBr by attosecond transient absorption spectroscopy. Science, 365(6448):79-83, 2019.

[12] Michael S Schuurman and Albert Stolow. Dynamics at conical intersections. Annual review of physical chemistry, 69:427-450, 2018.

[13] Valérie Blanchet, Marek Z Zgierski, Tamar Seideman, and Albert Stolow. Discerning vibronic molecular dynamics using time-resolved photoelectron spectroscopy. Nature, 401(6748):52-54, 
1999.

[14] Albert Stolow, Arthur E Bragg, and Daniel M Neumark. Femtosecond time-resolved photoelectron spectroscopy. Chemical Reviews, 104(4):1719-1758, 2004.

[15] Toshinori Suzuki. Femtosecond time-resolved photoelectron imaging. Annu. Rev. Phys. Chem., 57:555-592, 2006.

[16] Christer Z Bisgaard, Owen J Clarkin, Guorong Wu, Anthony MD Lee, Oliver Geßner, Carl C Hayden, and Albert Stolow. Time-resolved molecular frame dynamics of fixed-in-space $\mathrm{CS}_{2}$ molecules. Science, 323(5920):1464-1468, 2009.

[17] Paul Hockett, Christer Z Bisgaard, Owen J Clarkin, and Albert Stolow. Time-resolved imaging of purely valence-electron dynamics during a chemical reaction. Nature Physics, 7(8):612, 2011.

[18] John D Hybl, Allison W Albrecht, Sarah M Gallagher Faeder, and David M Jonas. Twodimensional electronic spectroscopy. Chemical physics letters, 297(3-4):307-313, 1998.

[19] David M Jonas. Two-dimensional femtosecond spectroscopy. Annual review of physical chemistry, 54(1):425-463, 2003.

[20] Shaul Mukamel. Multidimensional femtosecond correlation spectroscopies of electronic and vibrational excitations. Annual review of physical chemistry, 51(1):691-729, 2000.

[21] Robert W. Field. Spectra and Dynamics of Small Molecules. Springer International Publishing, 2015 .

[22] Peter M Felker. Rotational coherence spectroscopy: studies of the geometries of large gas-phase species by picosecond time-domain methods. The Journal of Physical Chemistry, 96(20):7844$7857,1992$.

[23] Albert Stolow and Jonathan G. Underwood. Time-resolved photoelectron spectroscopy of non-adiabatic dynamics in polyatomic molecules. Adv. Chem. Phys., 139:497, 2008.

[24] Jun John Sakurai and Jim Napolitano. Modern Quantum Mechanics, volume 261. Pearson, 2014 .

[25] Karl Blum. Density Matrix Theory and Applications, volume 64. Springer Science \& Business Media, 2012.

[26] Stuart C Althorpe and Tamar Seideman. Molecular alignment from femtosecond time-resolved photoelectron angular distributions: Nonperturbative calculations on NO. The Journal of Chemical Physics, 110(1):147-155, 1999.

[27] Jonathan G Underwood and Katharine L Reid. Time-resolved photoelectron angular dis- 
tributions as a probe of intramolecular dynamics: Connecting the molecular frame and the laboratory frame. The Journal of Chemical Physics, 113(3):1067-1074, 2000.

[28] Katharine L Reid and Jonathan G Underwood. Extracting molecular axis alignment from photoelectron angular distributions. The Journal of Chemical Physics, 112(8):3643-3649, 2000.

[29] Masaaki Tsubouchi, Benjamin J. Whitaker, Li Wang, Hiroshi Kohguchi, and Toshinori Suzuki. Photoelectron imaging on time-dependent molecular alignment created by a femtosecond laser pulse. Physical Review Letters, 86:4500-4503, May 2001.

[30] Arthur E Bragg, Roland Wester, Alison V Davis, Aster Kammrath, and Daniel M Neumark. Excited-state detachment dynamics and rotational coherences of $\mathrm{C}^{2-}$ via time-resolved photoelectron imaging. Chemical Physics Letters, 376(5-6):767-775, 2003.

[31] Masaaki Tsubouchi and Toshinori Suzuki. Photoelectron kinetic energy dependence in near threshold ionization of NO from a state studied by time-resolved photoelectron imaging. The Journal of Chemical Physics, 121(18):8846-8853, 2004.

[32] Yoshi-Ichi Suzuki and Toshinori Suzuki. Determination of ionization dynamical parameters by time-resolved photoelectron imaging. Molecular Physics, 105(11-12):1675-1693, 2007.

[33] Ying Tang, Yoshi-Ichi Suzuki, Takuya Horio, and Toshinori Suzuki. Molecular frame image restoration and partial wave analysis of photoionization dynamics of NO by time-energy mapping of photoelectron angular distribution. Physical Review Letters, 104(7):073002, 2010.

[34] Paul Hockett. General phenomenology of ionization from aligned molecular ensembles. New Journal of Physics, 17(2):023069, 2015.

[35] Claude Marceau, Varun Makhija, Dominique Platzer, A Yu Naumov, PB Corkum, Albert Stolow, DM Villeneuve, and Paul Hockett. Molecular frame reconstruction using time-domain photoionization interferometry. Physical Review Letters, 119(8):083401, 2017.

[36] Varun Makhija, Xiaoming Ren, Drue Gockel, Anh-Thu Le, and Vinod Kumarappan. Orientation resolution through rotational coherence spectroscopy. arXiv preprint arXiv:1611.06476, 2016.

[37] Péter Sándor, Adonay Sissay, François Mauger, Paul M Abanador, Timothy T Gorman, Timothy D Scarborough, Mette B Gaarde, Kenneth Lopata, Kenneth J Schafer, and Robert R Jones. Angle dependence of strong-field single and double ionization of carbonyl sulfide. Physical Review A, 98(4):043425, 2018. 
[38] Yoshi-ichi Suzuki, Mauro Stener, and Tamar Seideman. Multidimensional calculation of timeresolved photoelectron angular distributions: The internal conversion dynamics of pyrazine. The Journal of chemical physics, 118(10):4432-4443, 2003.

[39] Claude Marceau, Varun Makhija, Peng Peng, Marius Hervé, PB Corkum, A Yu Naumov, A Stolow, and DM Villeneuve. Non-born-oppenheimer electronic wave packet in molecular nitrogen at 14 ev probed by time-resolved photoelectron spectroscopy. Physical Review A, 99(2):023426, 2019.

[40] Albert Stolow and Jonathan G Underwood. Time-resolved photoelectron spectroscopy of non-adiabatic dynamics in polyatomic molecules. Advances in Chemical Physics, 139:497$583,2008$.

[41] Denitsa Baykusheva, Peter M Kraus, Song Bin Zhang, Nina Rohringer, and Hans Jakob Wörner. The sensitivities of high-harmonic generation and strong-field ionization to coupled electronic and nuclear dynamics. Faraday discussions, 171:113-132, 2014.

[42] Song Bin Zhang, Denitsa Baykusheva, Peter M Kraus, Hans Jakob Wörner, and Nina Rohringer. Theoretical study of molecular electronic and rotational coherences by high-orderharmonic generation. Physical Review A, 91(2):023421, 2015.

[43] Samuel G Walt, Niraghatam Bhargava Ram, Marcos Atala, Nikolay I Shvetsov-Shilovski, Aaron Von Conta, Denitsa Baykusheva, Manfred Lein, and Hans Jakob Wörner. Dynamics of valence-shell electrons and nuclei probed by strong-field holography and rescattering. Nature communications, 8:15651, 2017.

[44] Ruaridh Forbes, Varun Makhija, Kévin Veyrinas, Albert Stolow, Jason WL Lee, Michael Burt, Mark Brouard, Claire Vallance, Iain Wilkinson, Rune Lausten, et al. Time-resolved multimass ion imaging: Femtosecond uv-vuv pump-probe spectroscopy with the pimms camera. The Journal of Chemical Physics, 147(1):013911, 2017.

[45] Marcus Beutler, Masood Ghotbi, Frank Noack, and Ingolf Volker Hertel. Generation of sub50-fs vacuum ultraviolet pulses by four-wave mixing in argon. Optics letters, 35(9):1491-1493, 2010 .

[46] M Ghotbi, M Beutler, and F Noack. Generation of $2.5 \mu \mathrm{j}$ vacuum ultraviolet pulses with sub50 fs duration by noncollinear four-wave mixing in argon. Optics Letters, 35(20):3492-3494, 2010.

[47] ST Pratt. Photoionization dynamics of the $\tilde{B}^{1} E^{\prime \prime}$ state of ammonia. The Journal of Chemical 
Physics, 117(3):1055-1067, 2002.

[48] MNR Ashfold, RN Dixon, RJ Stickland, and CM Western. 2+ 1 mpi spectroscopy of $\tilde{B}^{1} E^{\prime \prime}$ state $\mathrm{NH}_{3}$ and $\mathrm{ND}_{3}$ : rotational analysis of the origin bands. Chemical Physics Letters, 138(2-3):201-208, 1987.

[49] Mike NR Ashfold, Richard N Dixon, N Little, RJ Stickland, and Colin M Western. The $\tilde{B}^{1} E^{\prime \prime}$ state of ammonia: Sub-doppler spectroscopy at vacuum ultraviolet energies. The Journal of Chemical Physics, 89(4):1754-1761, 1988.

[50] JM Allen, MNR Ashfold, RJ Stickland, and CM Western. The ${ }^{1} E^{\prime \prime}$ state of nh3: the jahn-teller effect revealed by infrared-optical double resonance. Molecular Physics, 74(1):49-60, 1991.

[51] James KG Watson. Jahn-Teller and $L$-uncoupling effects on rotational energy levels of symmetric and spherical top molecules. Journal of Molecular Spectroscopy, 103(1):125-146, 1984.

[52] Hong-Guang Duan, RJ Dwayne Miller, and Michael Thorwart. Impact of vibrational coherence on the quantum yield at a conical intersection. The journal of physical chemistry letters, $7(17): 3491-3496,2016$.

[53] Hong-Guang Duan and Michael Thorwart. Quantum mechanical wave packet dynamics at a conical intersection with strong vibrational dissipation. The journal of physical chemistry letters, $7(3): 382-386,2016$.

[54] J Biesner, L Schnieder, J Schmeer, G Ahlers, Xiaoxiang Xie, KH Welge, MNR Ashfold, and RN Dixon. State selective photodissociation dynamics of $\tilde{A}$ state ammonia. I. The Journal of Chemical Physics, 88(6):3607-3616, 1988.

[55] J Biesner, L Schnieder, G Ahlers, Xiaoxiang Xie, KH Welge, MNR Ashfold, and RN Dixon. State selective photodissociation dynamics of $\tilde{A}$ state ammonia. II. The Journal of Chemical Physics, 91(5):2901-2911, 1989.

[56] Richard N Zare. Angular Momentum: Understanding Spatial Aspects in Chemistry and Physics. John Wiley and Sons, 1988.

[57] Samuel Beaulieu, Antoine Comby, Alex Clergerie, Jérémie Caillat, Dominique Descamps, Nirit Dudovich, Baptiste Fabre, Romain Géneaux, François Légaré, Stéphane Petit, et al. Attosecond-resolved photoionization of chiral molecules. Science, 358(6368):1288-1294, 2017.

[58] Zhensheng Tao, Cong Chen, Tibor Szilvási, Mark Keller, Manos Mavrikakis, Henry Kapteyn, and Margaret Murnane. Direct time-domain observation of attosecond final-state lifetimes in photoemission from solids. Science, 353(6294):62-67, 2016. 
[59] J. M. Glownia, A. Natan, J. P. Cryan, R. Hartsock, M. Kozina, M. P. Minitti, S. Nelson, J. Robinson, T. Sato, T. van Driel, G. Welch, C. Weninger, D. Zhu, and P. H. Bucksbaum. Self-referenced coherent diffraction X-ray movie of Ångstrom- and femtosecond-scale atomic motion. Physical Review Letters, 117:153003, Oct 2016.

[60] Peter M Kraus, Michael Zürch, Scott K Cushing, Daniel M Neumark, and Stephen R Leone. The ultrafast x-ray spectroscopic revolution in chemical dynamics. Nature Reviews Chemistry, $2(6): 82,2018$. 